\title{
Syndrome de Williams: élastine, LIM-kinase 1 et les autres...
}

Le syndrome de Williams, WS (1/20 000 naissances), est un trouble complexe du développement décrit il y a 35 ans [1], qui associe habituellement une dysmorphie faciale caractéristique, une cardiopathie congénitale (sténose aortique supravalvulaire ou SVAS), un comportement jovial et une atteinte très spécifique des fonctions cognitives visuospatiales, associée en général à une déficience mentale plus globale. Le diagnostic est le plus souvent évoqué lors de la découverte de l'anomalie cardiaque (SVAS) qui est présente dans les trois quarts des cas, parfois devant une hypercalcémie, très inconstante. Chez les enfants sans cardiopathie, le diagnostic est habituellement porté plus tardivement devant le retard psychomoteur et la forme très évocatrice du visage: une grande bouche avec une lèvre inférieure charnue et éversée, des fentes palpébrales rétrécies par un abondant pannicule cutané péri-orbitaire, des joues pleines et une hypoplasie malaire. Le profil cognitif observé dans le WS comporte un déficit des fonctions visuospatiales, c'est-àdire la capacité de visualiser puis d'assembler des objets dans l'espace. Dans le WS, ce n'est pas la perception de la forme de l'objet qui est perturbée - ces sujets ont même une faculté hors du commun de reconnaître les visages - mais c'est l'organisation des objets dans l'espace qui est très altérée: devant une figure complexe ou un ensemble d'objets, les sujets atteints de WS sont attentifs uniquement aux détails, et sont incapables d'identifier et d'organiser ces détails en un ensemble cohérent [2]. En revanche, leur expression verbale est normale et comporte souvent un vocabulaire très élaboré pour l'âge. Ce profil neuropsychologique ressemble à celui de sujets atteints de lésions acquises

La localisation en 7q11.23 de l'anomalie génétique en cause dans les sténoses aortiques supravalvulaires familiales (SVAS), au niveau du locus de l'élastine (ELN), fut initialement suggérée par l'observation d'une famille atteinte d'une SVAS qui co-ségrégeait avec une translocation $\mathrm{t}(6: 7)$ (p21.1;q11.23); elle a été confirmée par une étude de liaison génétique dans deux autres familles de SVAS. Cette localisation des SVAS familiales isolées a donné l'idée au groupe de Mark Keating à Salt Lake City de rechercher par FISH (fluorescent in situ hybridization) et dosage génique utilisant le southern blot, une hémizygotie du gène $E L N$ chez 4 WS familiaux et 5 WS sporadiques [3]. Depuis, des délétions submicroscopiques emportant le gène $E L N$ ont été mises en évidence chez des centaines de WS, soit par FISH, soit par l'étude en PCR des contributions parentales pour des marqueurs hautement polymorphes de la région $7 q 11.23$. L'origine de la délétion est indifféremment d'origine paternelle $(40 \%)$ ou maternelle $(60 \%)$ et résulte dans la plupart des cas d'une recombinaison inégale lors de la méiose [4], ce qui explique le caractère sporadique de la grande majorité des WS. Les exceptionnels WS familiaux se transmettent selon un mode autosomique dominant.

A la lumière de ces résultats, on conçoit volontiers que la diminution de moitié de la synthèse de l'élastine dans la lamina des artères, la peau et les cordes vocales, soit à l'origine de la SVAS, de rides précoces et d'une voix rauque, décrits dans le WS. Cependant, l'absence d'expression du gène $E L N$ dans le cerveau et l'existence de mutations de ce gène associées à une SVAS isolée ne permettent pas d'attribuer à ce gène les autres signes du WS, en particulier les troubles neuropsychologiques. D'autres gènes contigus à celui de l'élastine sont donc probablement impliqués dans le phénotype du WS. C'est grâce à une remarquable approche multidisciplinaire, comportant une analyse clinique fine des troubles cognitifs présents dans le WS et l'exploitation de modèles murins, que le même groupe de Mark Keating a démontré le rôle de la LIM-kinase 1 (LIM-K1) dans la genèse des troubles visuospatiaux caractéristiques du WS [5]. Les auteurs ont étudié deux familles présentant un syndrome de Williams partiel, c'est-à-dire comportant le faciès caractéristique, les troubles cutanés et vasculaires et, sur le plan neuropsychologique, une atteinte franche et élective des fonctions visuospatiales mais avec un niveau intellectuel global normal. L'étude moléculaire de ces deux familles de WS sans retard mental a permis d'isoler deux délétions de petite taille (83 kb et $300 \mathrm{~kb}$ ) contrastant avec les délétions de plus de $500 \mathrm{~kb}$ habituellement retrouvées dans le WS classique. Après avoir établi un contig de cosmides couvrant $100 \mathrm{~kb}$ et incluant la plus petite délétion, les auteurs ont adopté une stratégie originale pour identifier le gène en cause : partant de l'observation de troubles d'apprentissage spatial chez des souris dont une protéine kinase a été invalidée $[6,7]$, ils ont synthétisé des oligonucléotides complémentaires de séquences conservées communes aux gènes des tyrosine kinases et ont effectué une PCR sur les clones d'ADN génomique de la région. C'est ainsi qu'ils ont amplifié puis cloné et séquencé un fragment de 315 pb totalement identique à une séquence du gène $L I M-K 1$, récemment identifié par un autre groupe [8]. Après isolement de l'ADNc complet à partir d'une banque de cerveau humain, les auteurs ont confirmé la localisation de ce gène en 7q11.23. L'hypothèse d'un 
autre gène de la région impliqué dans les troubles visuospatiaux du WS a été écartée par séquençage systématique de plus de $100 \mathrm{~kb}$ et recherche informatique d'exons potentiels (programmes GRAIL et BLAST). Le gène LIM-K1 (16 exons répartis sur $37 \mathrm{~kb}$, ARNm de 3,3 kb) est exprimé dans le cerveau foetal et adulte, en particulier dans le cortex cérebral et code pour une protéine kinase comportant des domaines LIM [5]. Ces domaines LIM en forme de paires de doigt de zinc, sont présents dans des protéines de développement chez la levure (lin-11, ruec-3) et les mammifères, par exemple Isl-1, un facteur de transcription du pancréas endocrine. L'invalidation du gène LIM-K1 par recombinaison homologue chez la souris permettra de vérifier et d'élucider son rôle dans les fonctions visuospatiales. Mais l'histoire ne s'arrête pas là ! Le retard mental $(\mathrm{QI}<70)$, retrouvé chez $60 \%$ des sujets atteints de WS, implique probablement un autre gène que $E L N$ et $L I M-K 1$ puisque les délétions limitées à ces deux gènes sont retrouvées dans des WS à QI global normal [5]. Par ailleurs, le retard statural qui est inconstant dans le WS, est plus fréquent et sévère quand la délétion est d'origine maternelle [9] ; cela suggère l'implication d'un quatrième gène, soumis cette fois à empreinte parentale. A suivre...

V.D.P.

1. Williams JCP, Barrett-Boyes BG, Lowe JB. Supravalvular aortic stenosis. Circulation 1961; 24: 1311-8. 2. Bellugi U, Bihrle A, Jernigan T, Trauner D, Doherty S. Neuropsychological, neurological, and neuroanatomical profile of Williams syndrome. Am J Med Genet 1990; 6 : 115-25.

3. Ewart A, Morris C, Atkinson D, Jin W, Sternes K, Spallone P, Stock AD, Leppert M, Keating M. Hemizygosity at the elastin locus in a develop- mental disorder, Williams syndrome. Nature Genet 1996 ; 5 : 11-6.

4. Dutly F, Schinzel A. Unequal interchromosomal rearrangements may result in elastin gene deletions causing the Williams-Beuren syndrome. Hum Mol Genet 1996; 5 : 1893-8.

5. Frangiskakis JM, Ewart A, Morris C, Mervis C, Bertrand J, Robinson B, Klein B, Ensing G, Everett L, Green E, Pröschel C, Gutowski N, Noble M, Atkinson D, Odelberg S, Keating M. LIM-kinase 1 hemizygosity implicated in impaired visuospatial constructive cognition. Cell 1996; 86: 59-69.

6. Grant GN, O'dell T, Karl K, Stein P, Soriano P, Kandel E. Impaired long-term potentialisation, spatial learning, and hippocampal development in fyn mutant mice. Science 1992; 258: 1903-10.

7. Abeliovich A, Paylor R, Chen C, Kim J, Wehner $\mathrm{M}$, Tonegawa S. PKC $\gamma$ mutant mice exhibit mild deficits in spatial and contextual learning. Cell $1993 ; 75$ : 1263-71

8. Mizumo K, Okano I, Ohashi K, Nunoue K, Kuma K, Miyata T, Nakamura T. Identification of a human cDNA encoding a novel protein kinase with two repeats of the LIM/double zinc finger motif. Oncogene 1994; 9 : 1605-12.

9. Pérez Jurado L, Peoples R, Kaplan P, Ben Hamel CJ, Francke U. Molecular definition of the chromosome 7 deletion in Williams syndrome and parent-of-origin effects on growth. Am J Hum Genet 1996 ; 59 : 781-92. 